\title{
Kits19TumorSegmentation with VNet
}

\author{
Junqiangchen
}

1207173174 eqq.com

\begin{abstract}
Vnet for segmentation tumor and kidney.Fist,preprocess the kidney and kidney tumor data,second,segmentating kidney progress split into two steps:Corse segmentation and fine segmentation.third, segmentation kidney tumor process split into two steps:2d segmentation and $3 d$ segmentation,
\end{abstract}

\section{1、Preprocess}

analyze the ct image,and get the slice thickness and window width and position.

\subsection{Preprocess Kidney}

keep kidney region into fixed size $(512,512,64)$ for Corse Kidney Segmentation. generate patch $(128,128,64)$ kidney image and mask for Corse Kidney Segmentation. keep Kidney region range image for fine Kidney Segmentation. generate patch $(128,128,64)$ kidney image and mask for fine Kidney Segmentation. save patch image and mask into csv file.

Split csv into training set and test set.

\subsection{Preprocess Kidney Tumor}

generate tumor image and mask for 2d Kidney Tumor Segmentation. generate tumor image and mask for 3d Kidney Tumor Segmentation. save tumor image and mask path into csv file.

Split csv into training set and test set

\section{2、Kidney segmentation}

\subsection{Corse Kidney Segmentation}

Corse Kidney Segmentation training:we use Vnet for segentation,input size is (128x128x64),learning rate is 0.001 ,optimizer is adam,epochs is 20 ,batchsize is 1 .

Corse Kidney Segmentation inference.

this step get Corse Kidney range,can find the start and end pos in the kidneyrang.txt

\subsection{Fine Kidney Segmentation}

Fine Kidney Segmentation training: we use Vnet for segentation,input size is (128x128x64),learning rate is 0.001 ,optimizer is adam,epochs is 20

Fine Kidney Segmentation inference.

this step following the 2.1 result,get fine Kidney result.

\subsection{Fine Kidney Segmentation}

remove Kidney Segmentation small object. 


\section{3、Tumor segmentation}

\subsection{2d Kidney Tumor Segmentation}

$2 d$ Kidney Tumor Segmentation training:we ues Vnet2d for segmeantion, input size is (512x512),learning rate is 0.001 ,optimizer is adam,epochs is 20 ,batchsize is 2 .

2d Kidney Tumor Segmentation inference

this step get $2 \mathrm{~d}$ slice tumor result.

\subsection{3d Kidney Tumor Segmentation}

3d Kidney Tumor Segmentation training: we use Vnet for segentation,input size is (128x128x64),learning rate is 0.001 ,optimizer is adam,epochs is 20 ,batchsize is 1 .

3d Kidney Tumor Segmentation inference

this step get $3 d$ tumor result

\subsection{Kidney Tumor Result Process}

remove Kidney Tumor Segmentation small object.

calculate overlap between $2 d$ tumor and $3 d$ tumor reslut.

save the region of $2 \mathrm{~d}$ tumor and $3 \mathrm{~d}$ tumor reslut that connect overlap region.

save the region of $2 d$ tumor and $3 d$ tumor within Kidney result.

merge the above two result and get final tumor result

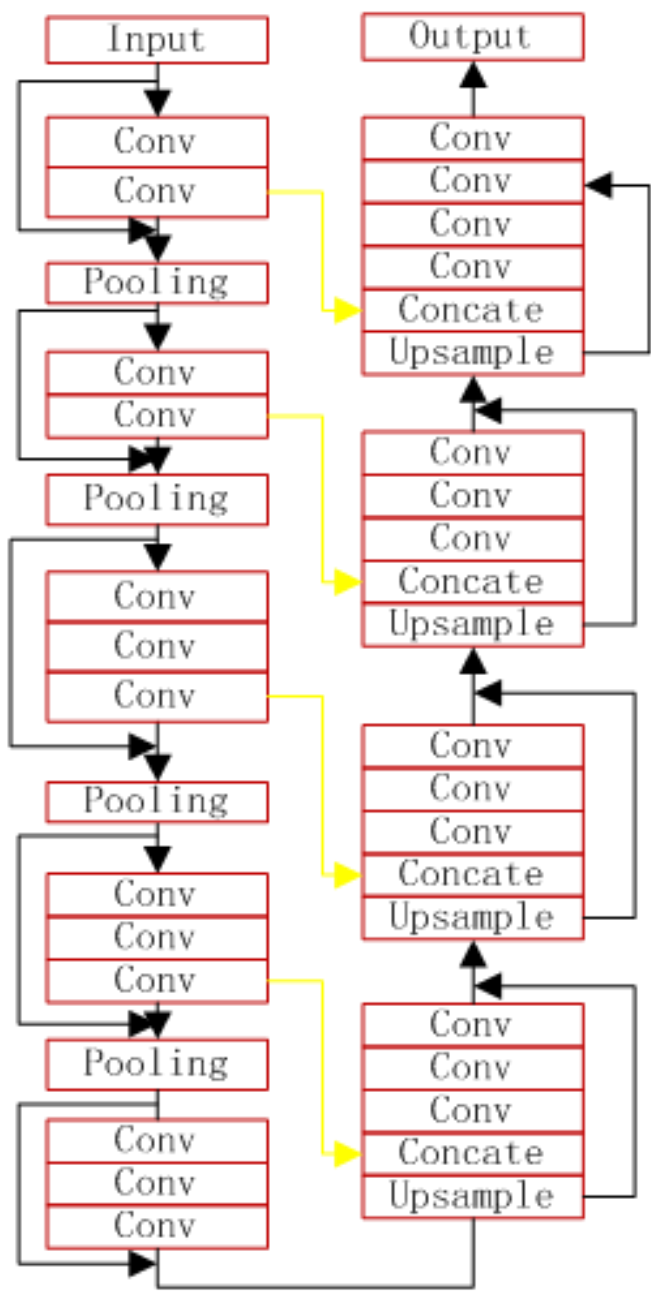


reference

You can find the source code on github: https://github.com/junqiangchen/KiTS19-Challege 